\title{
Parameter Optimization of Optical Sensor Calibration in Optical Coordinate Measuring System
}

\author{
Chengwei $\mathrm{Li}^{1,}$, Jingliang $\mathrm{Liu}^{1, \mathrm{~b}}$, Laipeng $\mathrm{Lv}^{1, \mathrm{c}}$, Bin Zhang ${ }^{1, \mathrm{~d}}$ and Jianguo \\ Fang ${ }^{1, e}$ \\ ${ }^{1}$ Aviation Key Laboratory of Science and Technology onPrecision Manufacturing NanYuan East \\ Road NO.5, FengTai District, Beijing, China \\ a391666866@qq.com, bj17519970@163.com, 'cvictorphantom@live.cn, 'zhangbin008007@163.com, \\ epanimal_123@163.com
}

Keywords: parameter optimization, optical coordinate measuring system, calibration method, optical sensor

Abstract. The different positions and angles of the optical sensor are calibrated before using the optical coordinate measuring system. In this paper, we analyze and optimize the common calibration process, reduce the unnecessary links, shorten the calibration time, and improve the efficiency of the optical coordinate measuring system by 50 percent.

\section{Introduction}

High efficiency and high accuracy are the improvement direction of the coordinate measuring[1]. With the development of industry, more processes need to be measured in order to solve the problem. In the process of machining, there will be the high temperature surface, the complex structure, the free surface, the soft surface during the machining process, which need to be measured on-line to ensure the machining accuracy. These different kinds of surfaces and structure also limit the use of the contact probe.

Recently, non-contact coordinate measuring system such as optical coordinate measuring system("OCMS" in short, shown in Figure 1) can achieve the measurement accuracy of $15 \mu \mathrm{m}$, which can fit the measuring requirement of machining process and some kind of work pieces with low accuracy requirement. With these characteristics, OCMS can be used in the intelligent production line as the measuring unit. Based on the OCMS, the optical sensor also can be positioned on the machining tools to measuring between the machining processes. All manuscripts must be in English, also the table and figure texts, otherwise we cannot publish your paper.

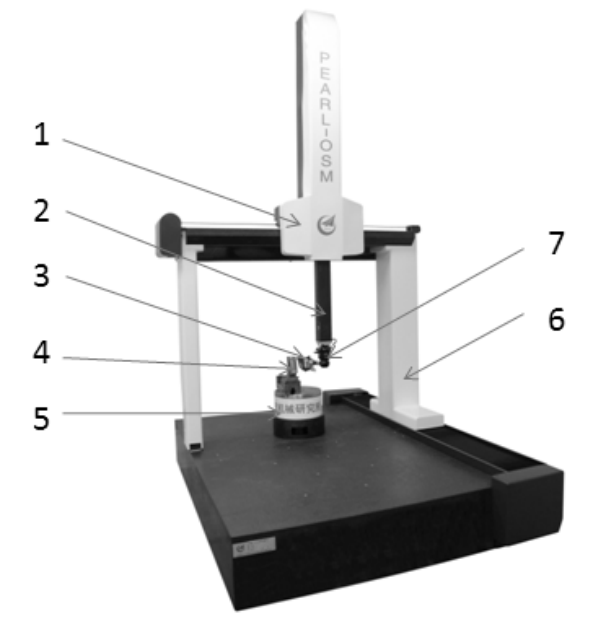

Figure 1 Optical Coordinate Measuring System

(1-Y axis, 2-Z axis, 3-optical sensor, 4-work piece, 5-rotary table, 6-X axis, 7-PH10M)

Before we use OCMS, not only the positions of optical sensor and the rotary table, but also the displacement caused by the rotary movement of PH10M should be calibrated (called "calibration" in 
total). Benefit from the scanning function of the optical sensor, OCMS can shorten the measuring time so that the efficiency of calibration will influence the efficiency of OCMS.

\section{Calibration Method}

The optical beam of the optical sensor is used as a one - dimensional space, and the position of the optical sensor is located at the origin of the optical beam. In the process of installation, the error vector (shown in figure 2) between origin of the optical beam and the position of the machine in the coordinate measuring system can be represented by a three space vector. The vector changes with the rotary movement of $\mathrm{PH} 10 \mathrm{M}$. If we need two or more angles during the measuring process, we should calibrate the error vector between these angles. To get the position of the spot, we also need the direction of the optical beam.

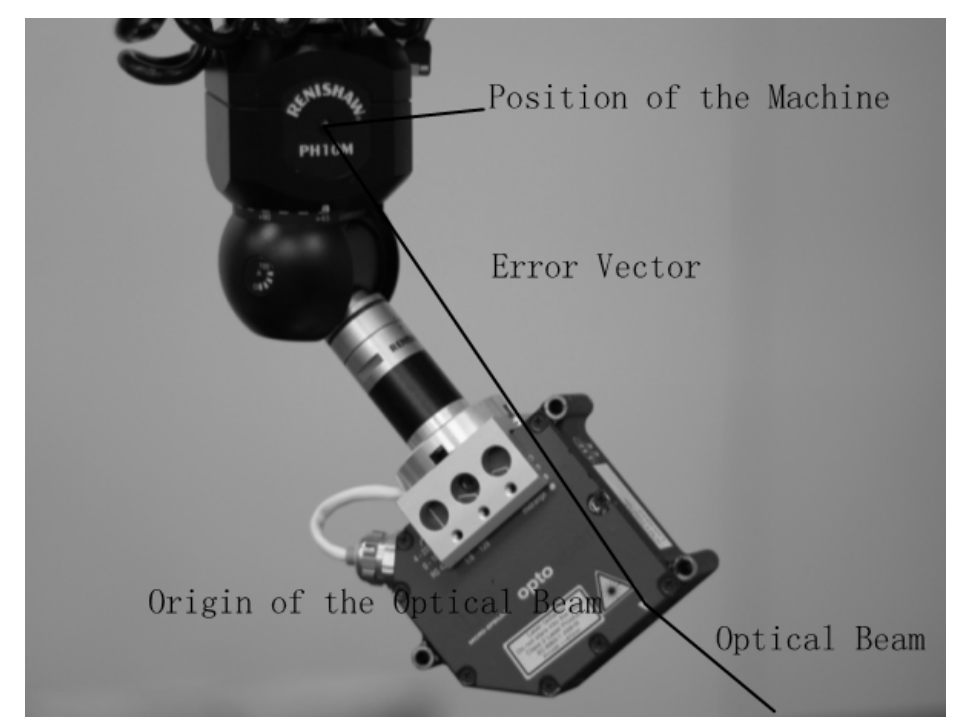

Figure 2 PH10M and optical sensor

When we measure symmetrical work piece, rotary table should be used. As a result, we should also know the position of the rotary table. This is the problem that answered by contact coordinate measuring system, so we won't discuss it in this paper.

Calibration of the Optical beam Direction. For the calibration of the optical beam direction, different calibration methods have been given by several experts[2]. Among these calibration methods, both the "Conjugate Pair Calibration Method" and the "Seven Points Calibration Method" use the standard ball as calibration objects, and both of them can meet the calibration requirements of all directions. According to the comparison of two kinds of calibration methods with the calibration requirements of high speed, high efficiency and high precision, the "Seven Points Calibration Method" is selected.

In the single optical beam direction, the influence of the error vector can be neglected. The position of the optical sensor spot can be expressed as $(x+l \cos \alpha, y+l \cos \beta, z+l \cos \lambda)$. Among them, $\mathrm{x}, \mathrm{y}$, $\mathrm{z}$ are the position of the OCMS, 1 is the output of the optical sensor and $\alpha, \beta, \gamma$ show the direction of the optical sensor axis. When the ball is used as the calibration object, a total of 7 unknowns are in the theoretical model shown in Figure 3. 


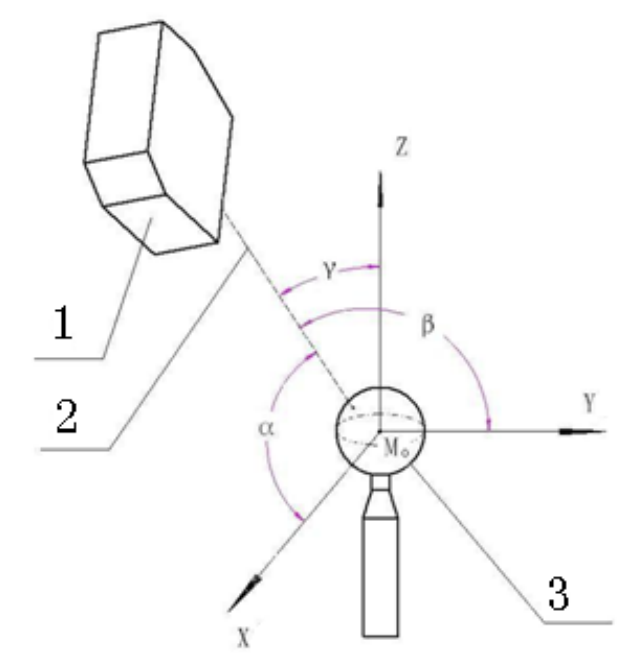

Figure 3 optical sensor beam calibration priciple

(1- optical sensor, 2-optical beam, 3-stanard ball)

Taking 7 groups of different measurement data, we can establish the following equations and solve.

$$
\left[x_{0}-\left(x_{i}+l_{i} \cos \alpha\right)\right]^{2}+\left[y_{0}-\left(y_{i}+l_{i} \cos \beta\right)\right]^{2}+\left[z_{0}-\left(z_{i}+l_{i} \cos \lambda\right)\right]^{2}=r^{2}
$$

In these equations, $i=1,2, \ldots, 7$, and $x i$, yi, zi, li mean the position of the OCMS in different equations and the output of the optical sensor at that place, and $\mathrm{x} 0, \mathrm{y} 0, \mathrm{z} 0$ mean the position of the standard ball's spherical center. We can also view $\left(x_{0}-l_{i} \cos \alpha, y_{0}-l_{i} \cos \beta, z_{0}-l_{i} \cos \lambda\right)$ as the spherical center of the ball that we reverse the standard ball by li along the optical beam. So if we achieve 4 different groups of data with the same sensor output, we can get the spherical center.

In order to obtain the optical beam direction, we need two spherical centers with different sensor output. We can obtain the radius in the first dummy standard ball of data with the same sensor output, so that we just need 3 groups of data for the second dummy standard ball. The direction of the optical beam can be obtained by solving the equation.

Calibration of Error Vector Caused by Rotation. By calibration the optical beam direction, we can measure the work piece from one angle. But almost all the work piece cannot be described from one side. So we must take the error vector in mind. With the rotation of the PH10M, the relative position between optical sensor and the measuring system changes. Therefore, we should measure the accuracy of the geometric objects to get relative position between the calibration object and the work piece. In this way, the calibration should also fit the requirement of all the angles that will be used. Moreover, we have a standard ball, so we choose the standard ball as the calibration object for the calibration of the error vector.

By measuring the standard ball from different angles, we can unify the measurement points under different angles into another coordinate system whose axis parallel to the machine coordinate system.

After all the calibrations of the optical sensor, the optical coordinate measuring system can be used to carry out the non-contact scanning measurement.

\section{Calibration Experiments}

Accuracy is the core content of the coordinate measuring system. If the precision can not meet the requirements, no matter how high the efficiency is useless. In two calibration processes, the standard ball are selected as the calibration object, and the key point of calibration is also measuring the spherical center of the standard ball in the machine coordinate calculation. Therefore, how to measure the spherical center of the standard ball quickly and accurately is important in the process of calibration. 
In the process of using the contact probe to measure the ball, the measuring error will decrease with the increase of the contact area of the measuring point (as shown in Figure 4). It can be seen from figure 4 that in order to improve the measurement accuracy, the coverage of the measurement points on the standard ball should be as large as possible.

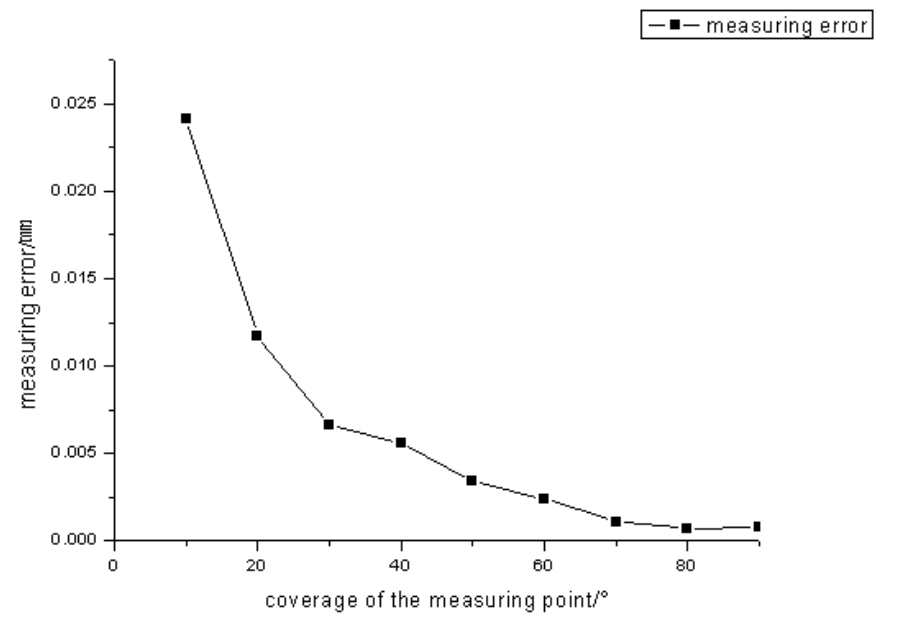

Figure 4 the relationship between coverage of the measuring point and the measuring error

The optical sensor used in this thesis is IDL1700--the single eye laser sensor. The incident angle characteristic is one of the key points that influence the accuracy of the laser sensor. Based on the experiments made by other experts, we calibrate the incident angle characteristic that are shown in Figure 50. From the figure 5, it can be seen that the measuring accuracy decreases with the increase of the incident angle between the optical beam and the surface normal.

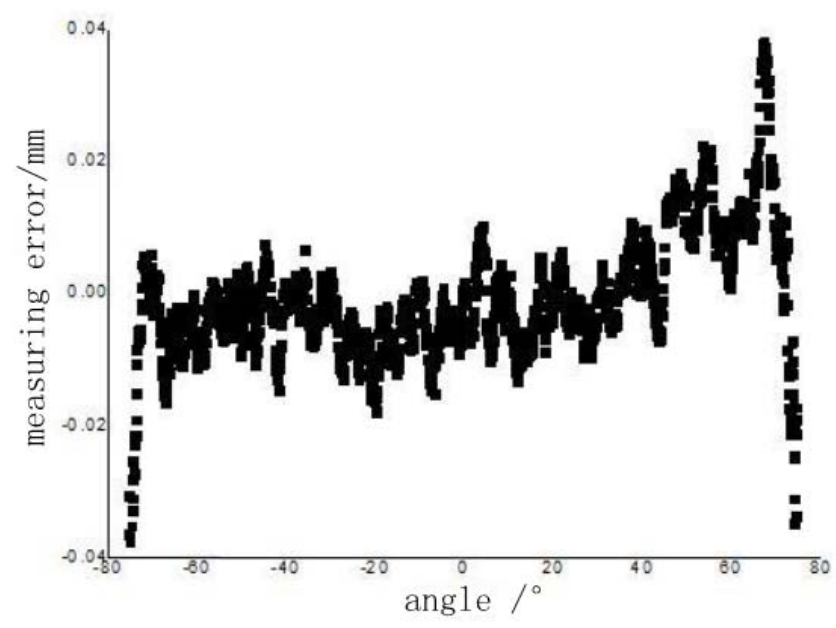

Figure 5 the relationship between incident angle and measuring error

Therefore, in order to ensure the accuracy, during the calibration, the coverage of the optical sensor spot should not be too large. To sum up, we need to select the appropriate coverage of the optical sensor spot, which can meet the accuracy requirements.

\section{Parameters Optimization of Calibration Experiment}

Parameters Optimization of Optical Beam Direction Calibration. In the process of optical beam calibration, we need 4 groups of data with the same optical sensor output and another 3 groups of data with the same output but different from the first output. When we get these data by handle, we seldom get the data both accurately and full covered the coverage. Therefore, we need to compile the calibration program to ask the control system show us the required calibration data by telling the controller the spherical center and the radius of the standard ball, and the coverage of the measuring 
points. In this way, the program controls OMCS for automatic calibration, in order to improve the calibration efficiency. The flow chart of calibration process is shown in figure 60:

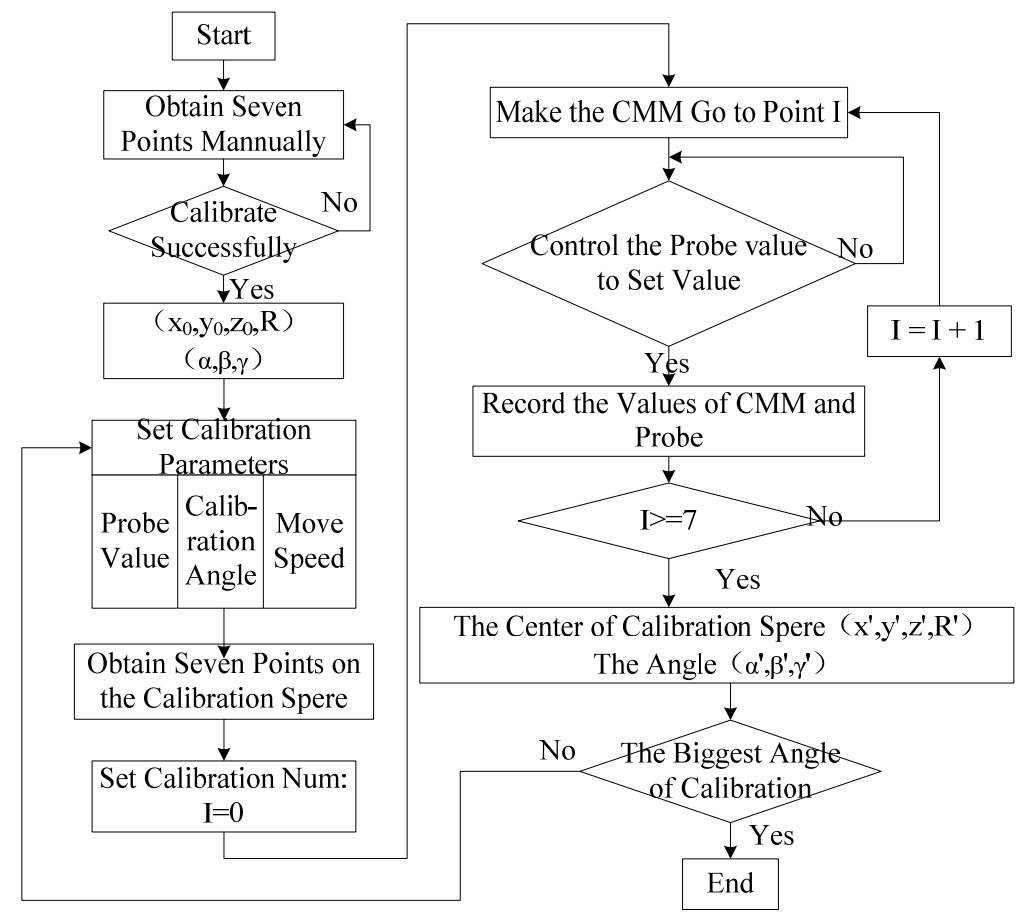

Figure 6 the flow chart of optical beam calibration

When we use the calibration program, we need to set he automatic calibration parameters. From Figure 5 above, it can be seen that when the coverage is more than 70 degrees, the incident angle characteristic will cause a large measurement error, and according to the actual situation, the coverage also dues to the area where the receiver of the optical sensor can get the diffuse reflection light. So the optical sensor spot coverage should not be greater than 60 degrees cone of the spherical center. And from Figure 3, when the coverage of the cone angle is less than 30 degrees, the measuring method will cause a large measurement error. In summary, the coverage of the optical beam calibration experiment should be at $30 \sim 60$ degrees.

To achieve the best coverage of the optical beam calibration, the parameters are divided into a single parameter of each 5 degrees in the range of $30 \sim 60$ degrees. The averages of each parameter's 10 groups of experimental results are obtained. In this experiment, the standard ball with a diameter of $49.995 \mathrm{~mm}$ is used to calibrate the optical beam. With these calibration results, the radius and the spherical center can be obtained by measuring the standard ball time after time. The mean radius of the standard ball and spot coverage are shown in Table 1.

Table 1 Relationship between spot coverage and the measuring mean radius

\begin{tabular}{|l|l|}
\hline coverage $\left({ }^{\circ}\right)$ & mean radius $(\mathrm{mm})$ \\
\hline 30 & 49.986 \\
\hline 35 & 49.985 \\
\hline 40 & 49.988 \\
\hline 45 & 49.993 \\
\hline 50 & 49.994 \\
\hline 55 & 49.995 \\
\hline 60 & 49.989 \\
\hline
\end{tabular}

From the data in the table above, it can be seen that the measurement error is less than $10 \mu \mathrm{m}$ in the range of 40 60 degrees. And the precision can meet the requirement of the OCMS. 
Parameters Optimization of Error Vector Calibration. Based on the experimental results above, the coverage of the optical sensor spot should be in the range of 40 60 degrees. When different optical beam directions are used in measuring process, error vector caused by rotation of PH10M should be calibrated.

After calibration of the optical beam direction, we can measure the standard ball to get the spherical center. In this way, we can get the relationship between the measuring point on the work piece and the spherical center of the standard ball. We measure the standard ball every time after we calibrate a new optical beam direction, and the measuring points in this direction can be translated into the reference frame whose axis parallel the axis of the machine coordinate and whose origin is the spherical center of the standard ball. In this calibration process, the coverage of the spots on the standard ball is the key parameter.

In the range of 40 60 degrees, the error vector calibration experiments are divided into 5 groups. Each group we measure 10 points under 5 optical beam directions. With 10 measuring points, the radius of the standard ball can be fitted, and the fitting results are shown in Table 2:

Table 2 Relationship between coverage and measuring radius

\begin{tabular}{|c|c|}
\hline coverage $\left({ }^{\circ}\right)$ & measuring radius $(\mathrm{mm})$ \\
\hline 40 & 49.999 \\
\hline 45 & 50.008 \\
\hline 50 & 50.004 \\
\hline 55 & 49.984 \\
\hline 60 & 49.991 \\
\hline
\end{tabular}

Through the results of the experiments, it can be seen that the measuring error of the error vector calibration from 40 degrees to 60 degrees is less than $10 \mu \mathrm{m}$. So the calibration method can meet the requirements of the OCMS.

\section{Conclusions}

According to the experiment results, the calibration accuracy of the two calibration methods can meet the requirements the optical coordinate measuring system. In the process of preparing, we just need to expend the coverage to 40 degree. In the range of $40 \sim 60$ degrees, it is not obvious to improve the accuracy of the calibration, but it will increase the calibration time and reduce the efficiency obviously.

In summary, in the course of measurement preparing, the commendatory optical sensor spot coverage is 40 degree.

\section{Acknowledgement}

The authors are grateful for the financial support provided by the National Science and Technology Major Project(2014ZX04001021-7)

\section{References}

[1] Guoxiong Zhang, The Development of Coordinate Measuring Technology. [J] Infrared and Laser Engineering, 2008 April, Vol. thirty-seventh, suppl, in Chinese.

[2] David Bunimovich, Calibration Process for Shape Measurement: U.S,6,199,024 B1[P].2001-03-06.

[3]Zexiao Xie, The Calibration method of non-contact probe based on Vision. China, 200510044577.3[P].2006-03-01, in Chinese 
[4]Huicheng Zhou, Lizhan Zeng, Jihong Chen. The Calibration of the Laser Sensor's Beam Direction.[J]. Chinese Journal of Scientific Instrument, 2004,25(3)388-391, in Chinese.

[5]Keqing Lu, Wen Wang, Zichen Chen. Calibration of Laser Sensor Beam Direction[J]. Oprics and Precision Engineering, 2010, 14(4)880-886, in Chinese.

[6]Yong Liu, Chao Bi, Ying Gao, et al. Research and application of plane calibration method of optical sensor beam direction[J]. Measurement and Control Technology, 2013,32(Suppl):513-515, in Chinese.

[7]Jianguo Fang, Tan Zhang, Liang Fang. A Calibration Method of the Spot-Structured Pptical Probe's Spatial Position and Vector. China, 201310493454.2. in Chinese.

[8]Chengwei Li. Error Analysis and Calibration Methods of the Optical Coordinate Measuring System: [D].Beijing: China Aeronautical Establishment, 2014.

[9]Jingliang Liu, Yong Zhu, Zhenya Kang, et al. Study on Fast Calibration Model for Beam's Space Position of Point-Strctured Optical Probe. 2014 International Conference on Mechatronics Engineering and Modern Technologies in Industrial Engineering[A]. 